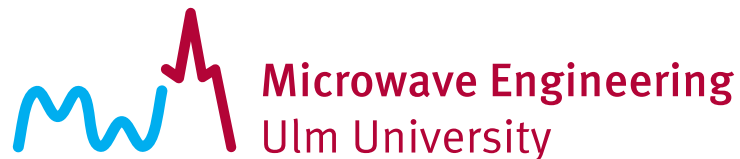

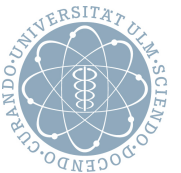

\section{Self-Aligning and Flexible Dielectric Waveguide Plug for MMICs at G-Band}

\author{
Martin Geiger, Martin Hitzler, Winfried Mayer, and Christian Waldschmidt
}

(C) 2020 IEEE. Personal use of this material is permitted. Permission from IEEE must be obtained for all other uses, in any current or future media, including reprinting/republishing this material for advertising or promotional purposes, creating new collective works, for resale or redistribution to servers or lists, or reuse of any copyrighted component of this work in other works. 


\title{
Self-Aligning and Flexible Dielectric Waveguide Plug for MMICs at G-Band
}

\author{
Martin Geiger, Student Member, IEEE, Martin Hitzler, Member, IEEE, Winfried Mayer, Senior Member, IEEE, \\ and Christian Waldschmidt, Senior Member, IEEE
}

\begin{abstract}
The packaging technology for transitions to dielectric waveguides in the frequency range above $100 \mathrm{GHz}$ is complex, must be highly precise, and the waveguides are usually permanently connected. This paper presents a transition from an MMIC to a flexible dielectric waveguide at G-Band $(140 \mathrm{GHz}$ to $220 \mathrm{GHz}$ ), which is self-aligning and thus reduces the requirements for packaging accuracy. Furthermore, the transition is mechanically decoupled to avoid mechanical stress to the MMIC and to reconnect it arbitrarily often. A patch radiator on a quartz glass carrier is excited by a coupler on the MMIC. It feeds the $\mathrm{HE}_{11}$ mode into a rigid, high-permittivity dielectric dome, which increases the coupling efficiency. The flexible dielectric waveguide is placed above the dome and fixed with Rohacell half shells. The minimum insertion loss measured with a BEOL MMIC is $3.0 \mathrm{~dB}$ at $168 \mathrm{GHz}$
\end{abstract}

Index Terms-dielectric waveguide, mechanical decoupling, MMIC, MMIC to dielectric waveguide transition, self-alignment.

\section{INTRODUCTION}

Large bandwidths and low-cost $\mathrm{SiGe}$ monolithic microwave integrated circuits (MMIC) make the frequency range above $100 \mathrm{GHz}$ attractive for both communication and radar. With flexible, low-loss dielectric waveguide interconnects, mmwave signals can be transmitted over long distances for boardto-board links [1] or to feed remote antennas [2], [3]. This opens up new fields of applications.

For this reason, several transitions from an MMIC to a dielectric waveguide have already been proposed in the literature for communication links [4]-[10]. Some transitions are based on resonant structures like dielectric resonators [4] or patches [5] to feed the dielectric waveguide. Another transition excites a wave in the dielectric waveguide with a half-mode substrate integrated waveguide [6]. In order to couple the signal more efficiently into the dielectric waveguide, the used materials have a high permittivity, such as high-resistivity silicon $\left(\varepsilon_{r}=11.9\right)$. Furthermore, the materials are low-loss, rigid, and in permanent contact with the MMIC, as required for short-range chip-to-chip interconnects.

Manuscript received November 12, 2019; revised December 16, 2019 accepted January 15, 2020.

This work was supported by the Ministry for Science, Research and Arts Baden-Württemberg, Germany, within the project ZAFH MikroSens. (Corresponding Author: Martin Geiger.)

M. Geiger and C. Waldschmidt are with the Institute of Microwave Engineering, Ulm University, 89081 Ulm, Germany (e-mail: martin-2.geiger@uniulm.de). M. Hitzler and W. Mayer are with Endress + Hauser GmbH + Co. KG, 79689 Maulburg, Germany.

Color versions of one or more of the figures in this letter are available online at http://ieeexplore.ieee.org.

Digital Object Identifier xxx

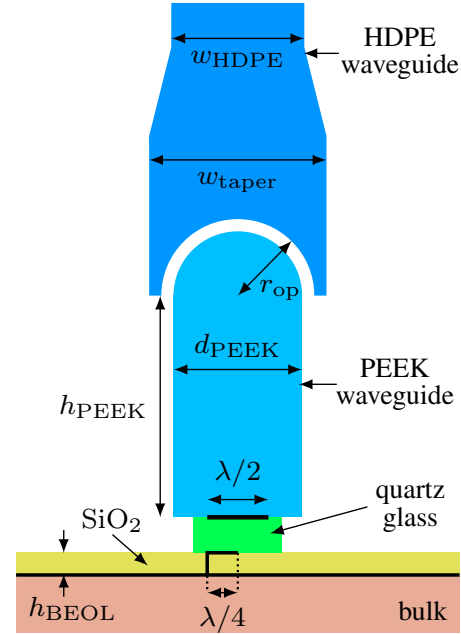

(a)

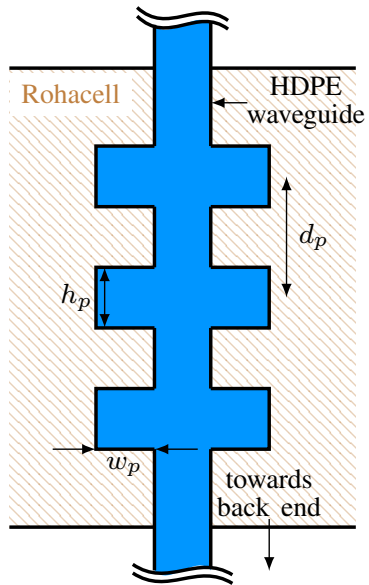

(b)
Fig. 1. Schematic cross-section of the transition from MMIC to (a) dielectric waveguide and (b) the Rohacell holder.

For applications with a signal transmission over several meters, the requirements for the transition and the dielectric waveguide are different. Due to the non-static scenario, a flexible waveguide made of Teflon or high-density polyethylene (HDPE) with a relative permittivity around 2.2 is necessary. Furthermore, a mechanical decoupling of MMIC and waveguide increases the transition robustness and allows to reconnect MMIC and waveguide several times. This can be realized by radiating into the dielectric waveguide [11], [12], with metallic waveguide adapters [13], or additional transitions to the PCB [14]. However, all approaches increase the insertion loss from the MMIC to the dielectric waveguide compared to the previously mentioned transitions.

This paper presents a mechanically decoupled transition from an MMIC to a flexible dielectric waveguide using a high-permittivity dielectric waveguide adapter to increase the coupling efficiency. Furthermore, the flexibility of the HDPE waveguide is utilized to align itself. The transition was designed and measured for a SiGe back end of line (BEOL) MMIC. In the following section, the transition principle is explained. In Section III the measurements results are shown and compared to state-of-the-art designs.

\section{TRANSITION PRINCIPLE}

The transition from the $\mathrm{SiO}_{2}$ back end $\left(\varepsilon_{r}=4, \tan \delta=0.02\right.$, $\left.h_{\mathrm{BEOL}}=10 \mu \mathrm{m}\right)$ to the flexible dielectric waveguide is composed of several components as shown in Fig. 1a. 


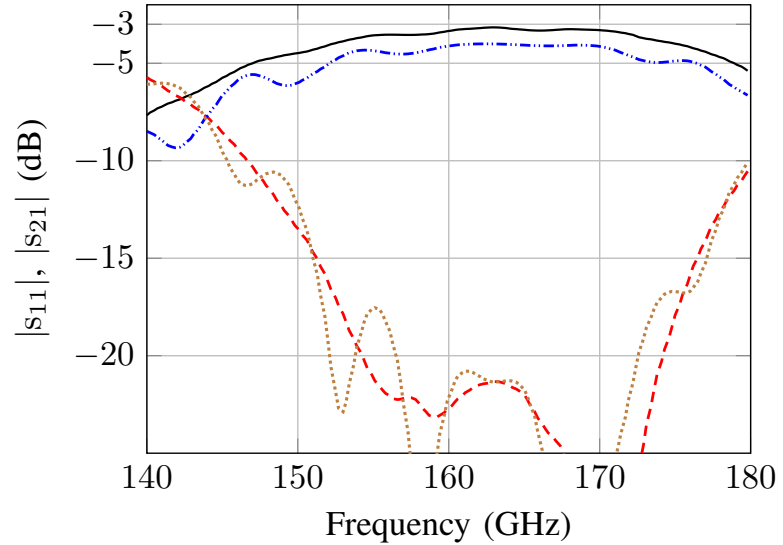

Fig. 2. Simulated S-Parameters for the transition from the back end to the flexible waveguide without holder $\left(\left|\mathrm{s}_{21}\right|(-),\left|\mathrm{s}_{11}\right|(---)\right)$ and with holder $\left(\left|\mathrm{s}_{21}\right|(-\cdots-\cdots),\left|\mathrm{s}_{11}\right|(\cdots \cdots \cdots)\right)$.

In order to decouple the field efficiently of the back end, a $\lambda / 2$-patch on a $100 \mu \mathrm{m}$ thick quartz glass carrier $\left(\varepsilon_{r}=3.8\right.$, $\tan \delta=0.001)$ is placed above a shortened $\lambda / 4$-patch, which is fed by a microstrip line on the back end [15], (cf. Fig. 3b). The length $l_{\text {patch }}$ of the $\lambda / 2$-patch determines the center frequency $f_{0}$ of the transition. With a back end area of only $170 \mu \mathrm{m} \times 220 \mu \mathrm{m}=0.01 \lambda_{0}^{2}$ the transition has a very compact design, with $\lambda_{0}$ corresponding to the wavelength at $f_{0}$.

On top of the patch a round dielectric waveguide dome with the diameter $d_{\mathrm{PEEK}}=780 \mu \mathrm{m}$ is placed, in which the $\mathrm{HE}_{11}$ mode is excited. By increasing the permittivity of the dielectric waveguide, the field is decoupled more efficiently of the back end. The dielectric waveguide is made of polyetheretherketone (PEEK), since it is very simple to machine. Furthermore, the permittivity of $\varepsilon_{r}=3.2(\tan \delta=0.006)$ is high enough to make the decoupling of the back end more efficient compared to $\operatorname{HDPE}\left(\varepsilon_{r}=2.25, \tan \delta=3.1 \cdot 10^{-4}\right)$.

A self-aligning transition is used to connect the flexible and mechanically decoupled dielectric waveguide. The round waveguide dome is spherically rounded with a hemisphere. The counterpart in the flexible waveguide has a spherical opening with a radius $r_{\mathrm{op}}$ of $490 \mu \mathrm{m}$, aligning itself in case of misalignment or mechanical stress. The hemispherical shapes serve not only for mechanical alignment but also for improved impedance matching. In order to avoid reflections at the interface, the wave impedance of the two dielectric waveguides is chosen to be very similar. The permittivity as well as the dimensions are the adjustable parameters. The flexible waveguide is made of HDPE and has a rectangular crosssection of $648 \mu \mathrm{m} \times 1295 \mu \mathrm{m}$. A tapering to a cross-section of $950 \mu \mathrm{m} \times 1295 \mu \mathrm{m}$ decreases the insertion loss of the transition and avoids additional radiation losses. Full wave simulations of the transition from the back end to the flexible waveguide result in a minimum simulated insertion loss of $3.1 \mathrm{~dB}$ with a $10 \mathrm{~dB}$ return loss bandwidth of $34 \mathrm{GHz}$ as shown in Fig. 2 . The losses are mainly caused by dielectric and metallic losses on the MMIC (70\%) and only $23 \%$ by radiation losses.

The requirements for the positioning precision of the quartz glass are high and it should be positioned with $50 \mu \mathrm{m}$ ac-
TABLE I

DIMENSIONS FOR THE TRANSITION IN $\mu \mathrm{m}$.

\begin{tabular}{c|c|c|c|c|c|c}
$w_{\text {patch }}$ & $l_{\text {patch }}$ & $h_{\text {PEEK }}$ & $w_{\text {taper }}$ & $h_{p}$ & $w_{p}$ & $d_{p}$ \\
\hline \hline 260 & 360 & 1000 & 950 & 750 & 1000 & 1750 \\
\hline
\end{tabular}

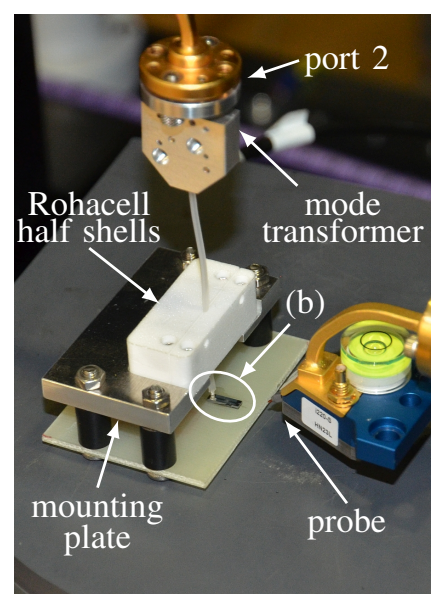

(a)

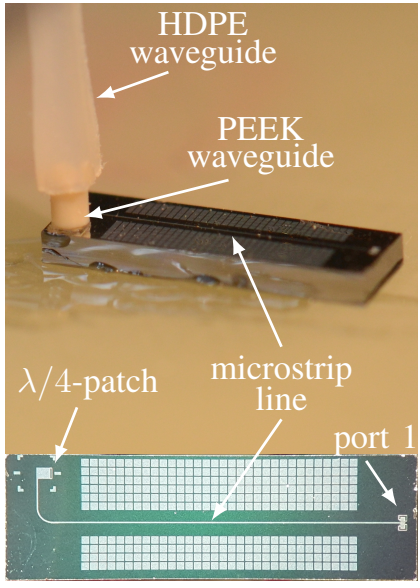

(b)
Fig. 3. Realized transition from the back end to the flexible dielectric waveguide (a) in the measurement setup with Rohacell holder and (b) a detailed view on the transition and the BEOL layout.

curacy. The pluggable interface between the dome and the flexible front end, however, is very robust against positioning tolerances due to the non-resonant coupling. A vertical displacement of $0.1 \mathrm{~mm}$ results in a larger air gap between the waveguide and the dome and reduces the transmission coefficient by $0.3 \mathrm{~dB}$. Displacements in horizontal direction or slight tilting are even less critical.

Therefore, a Rohacell shell $\left(\varepsilon_{r}=1.05, \tan \delta=0.005\right)$ is used to fix the flexible waveguide $1 \mathrm{~cm}$ above the BEOL MMIC. It disturbs the field distribution minimally and fits positively on a pin-shaped profile on the dielectric waveguide surface as shown in Fig. 1b. The pins fix the waveguide mechanically, but cause additional radiation losses. This increases the insertion loss by approximately $0.9 \mathrm{~dB}$ (Fig. 2) on average. The transition dimensions are listed in Table I.

\section{Transition Assembly and Measurements}

A BEOL MMIC was fabricated to characterize the transition. The quartz glass was metallized with a $2 \mu \mathrm{m}$ thick aluminum layer in a clean room process and glued on the BEOL MMIC with epoxy adhesive. The PEEK waveguide was fixed on top with epoxy adhesive. The flexible waveguide was clamped with two Rohacell half shells and aligned over the PEEK waveguide using an aluminum mounting plate and screws. The realized transition is shown in Fig. 3.

For the S-parameter measurements the BEOL MMIC was contacted with a probe, and the flexible dielectric waveguide was connected via a mode converter with a metallic waveguide. The S-parameters of the transition are determined by de-embedding the measured losses of the microstrip line $(6.9 \mathrm{~dB} \hat{=} 1 \mathrm{~dB} / \mathrm{mm})$, the mode converter $(0.5 \mathrm{~dB})$ [2], and the flexible dielectric waveguide $(0.3 \mathrm{~dB})$, since the calibration 
TABLE II

COMPARISON OF STATE-OF-THE-ART DESIGNS FOR TRANSITIONS FROM CHIP TO DIELECTRIC WAVEGUIDE.

\begin{tabular}{|c||c|c|c|c|c|c|}
\hline Ref. & Transition Design & Area $\left(\lambda_{0}^{2}\right)$ & Substrate, Height & $\begin{array}{c}\text { Frequency (GHz) } \\
\text { [rel. Bandwidth] }\end{array}$ & $\begin{array}{c}\text { Permittivity } \\
\text { Waveguide }\end{array}$ & IL (dB) \\
\hline \hline$[4]$ & self-aligned resonator & 0.028 & $\mathrm{BCB}\left(\varepsilon_{r}=2.55\right), 24 \mu \mathrm{m}$ & $100[7.2]$ & 9.8 & 2.15 \\
\hline$[5]$ & open-ended microstrip feed & 0.07 & $\mathrm{BCB}, 20 \mu \mathrm{m}$ & $175[12]$ & 11.9 & 3.2 \\
\hline$[6]$ & half-mode SIW & 0.189 & $\mathrm{SiO}_{2}, 10 \mu \mathrm{m}$ & $275[18.2]$ & 6.15 & 4.8 \\
\hline$[11]$ & $\begin{array}{c}\text { bow-tie dipole with metal } \\
\text { reflector }\end{array}$ & 0.07 & $\mathrm{SiO}_{2}, 10 \mu \mathrm{m}$ & $120[15]$ & 2.1 & 11 \\
\hline$[14]$ & $\begin{array}{c}\text { SIW on PCB fed by } \\
\text { flip-chip }\end{array}$ & - & $\begin{array}{c}\text { Megtron6 }\left(\varepsilon_{r}=3.6\right), \\
300 \mathrm{um}\end{array}$ & $120[25]$ & 2.1 & 3.4 \\
\hline $\begin{array}{c}\text { This } \\
\text { work }\end{array}$ & $\begin{array}{c}\text { shortened } \lambda / 4-p a t c h \\
\text { feeding a stacked design }\end{array}$ & 0.01 & $\mathrm{SiO}_{2}, 10 \mu \mathrm{m}$ & $160[18.8]$ & 2.25 & 3.0 \\
\hline
\end{tabular}

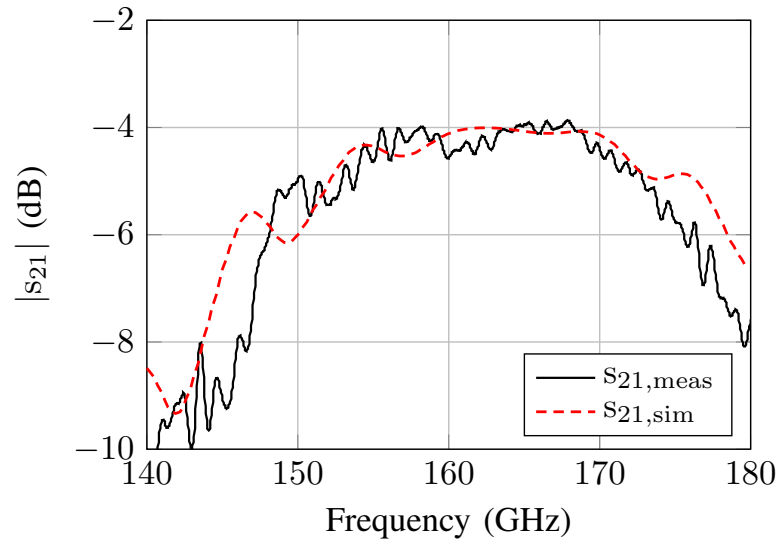

Fig. 4. Measured and simulated transmission coefficient $\left|\mathrm{s}_{21}\right|$.

reference plane was at the probe tip $\left(\mathrm{s}_{11}\right)$ or the waveguide interface $\left(\mathrm{s}_{21}, \mathrm{~s}_{22}\right)$.

The simulated and measured transmission coefficients are shown in Fig. 4. The maximum measured transmission coefficient is $-3.9 \mathrm{~dB}$ at $168 \mathrm{GHz}$. In the frequency range from $147 \mathrm{GHz}$ to $177 \mathrm{GHz}$ the transmission coefficient declines by $3 \mathrm{~dB}$ compared to the measured maximum. The measured values agree very well with the simulations. According to the simulations the Rohacell form has an insertion loss of $0.9 \mathrm{~dB}$. De-embedding this loss increases the transmission coefficient of the transition to $-3.0 \mathrm{~dB}$.

On the probe side, the measured reflection coefficient $\left|\mathrm{s}_{11}\right|$ is below $-10 \mathrm{~dB}$ in the frequency range from $149 \mathrm{GHz}$ to $179 \mathrm{GHz}$ as shown in Fig. 5. The reflection coefficient towards the waveguide is below $-10 \mathrm{~dB}$ for the complete measured frequency range. Simulations and measurements agree well.

\section{COMPARISON WITH STATE-OF-THE-ART DESIGNS}

The comparison of the proposed transition to state-of-the-art designs must not only consider the insertion loss (IL) and chip area, but also the mechanical construction, material properties, and the realization of the BEOL MMIC. Several transitions are listed in Table II and subdivided into rigid dielectric waveguides with high permittivity [4]-[6] and flexible, lowpermittivity waveguides [11], [14].

The presented transition has the lowest area consumption with a very small guard area (patch size plus $20 \mu \mathrm{m}$ ) around the

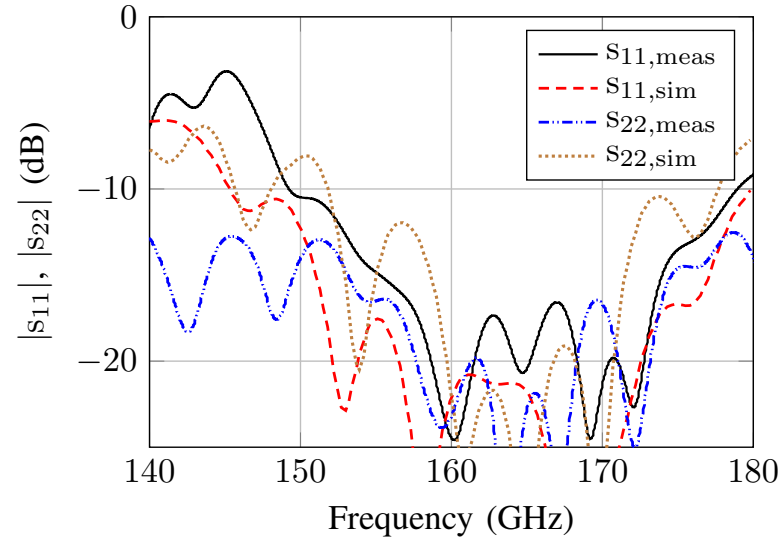

Fig. 5. Measured and simulated reflection coefficients $\left|s_{11}\right|$ (towards probe) and $\left|s_{22}\right|$ (towards rectangular waveguide).

patch and is fabricated with a commercial available IC process like [6], [11], [14]. With a relative bandwidth of $18.8 \%$ the transition is very broadband. The measured insertion loss of $3 \mathrm{~dB}$ is comparatively low. Transitions with comparable or lower insertion losses use higher low-loss substrates and highpermittivity dielectric waveguides, which improves the transition performance. Furthermore, the presented transition is mechanically decoupled, can be reconnected several times, and is even self-aligning, reducing the requirements concerning the positioning accuracy.

\section{CONClusion}

In this paper, a transition from MMIC to a flexible dielectric waveguide in the G-Band is presented. The transition is mechanically decoupled and aligns itself in case of slight positioning tolerances.

The transition is based on a stacked design made out of several components. A resonant $\lambda / 2$-patch, excited by the MMIC, feeds the fundamental $\mathrm{HE}_{11}$ mode into a rigid dielectric waveguide. By rounding the rigid waveguide end spherically, the flexible waveguide can be aligned with the corresponding counterpart above it. Rohacell half shells are used to fix the flexible waveguide. The minimum measured insertion loss without the Rohacell holders is $3 \mathrm{~dB}$ at $168 \mathrm{GHz}$. The transition is matched better than $10 \mathrm{~dB}$ over a bandwidth of $30 \mathrm{GHz}$. 


\section{REFERENCES}

[1] S. Fukuda, Y. Hino, S. Ohashi, T. Takeda, H. Yamagishi, S. Shinke, K. Komori, M. Uno, Y. Akiyama, K. Kawasaki, and A. Hajimiri, "A $12.5+12.5 \mathrm{~Gb} / \mathrm{s}$ Full-Duplex Plastic Waveguide Interconnect," IEEE Journal of Solid-State Circuits, vol. 46, no. 12, pp. 3113-3125, Dec. 2011.

[2] M. Geiger, M. Hitzler, J. Iberle, and C. Waldschmidt, "A Dielectric Lens Antenna Fed by a Flexible Dielectric Waveguide At $160 \mathrm{GHz}$," in 11th European Conference on Antennas and Propagation (EuCAP), Mar. 2017, pp. 3380-3383.

[3] M. Geiger and C. Waldschmidt, "160-GHz Radar Proximity Sensor With Distributed and Flexible Antennas for Collaborative Robots," IEEE Access, vol. 7, pp. 14977-14 984, 2019.

[4] U. Dey and J. Hesselbarth, "Building Blocks for a Millimeter-Wave Multiport Multicast Chip-to-Chip Interconnect Based on Dielectric Waveguides," IEEE Transactions on Microwave Theory and Techniques, vol. 66, no. 12, pp. 5508-5520, Dec. 2018.

[5] B. Yu, Y. Ye, X. Ding, Y. Liu, Z. Xu, X. Liu, and Q. J. Gu, "OrthoMode Sub-THz Interconnect Channel for Planar Chip-to-Chip Communications," IEEE Transactions on Microwave Theory and Techniques, vol. 66, no. 4, pp. 1864-1873, Apr. 2018.

[6] J. W. Holloway, L. Boglione, T. M. Hancock, and R. Han, "A Fully Integrated Broadband Sub-mmWave Chip-to-Chip Interconnect," IEEE Transactions on Microwave Theory and Techniques, vol. 65, no. 7, pp. 2373-2386, Jul. 2017.

[7] B. Yu, Y. Liu, Y. Ye, X. Liu, and Q. J. Gu, "Low-loss and Broadband GBand Dielectric Interconnect for Chip-to-Chip Communication," IEEE Microwave and Wireless Components Letters, vol. 26, no. 7, pp. 478480, Jul. 2016.

[8] B. Yu, Y. Liu, Y. Ye, J. Ren, X. Liu, and Q. J. Gu, "High-Efficiency Micromachined Sub-THz Channels for Low-Cost Interconnect for Planar Integrated Circuits," IEEE Transactions on Microwave Theory and Techniques, vol. 64, no. 1, pp. 96-105, Jan. 2016.

[9] U. Dey and J. Hesselbarth, "Millimeter-Wave Multicast Chip-to-Chip Interconnect Network Using Dielectric Slab Waveguide," in 2018 IEEE/MTT-S International Microwave Symposium - IMS, Jun. 2018, pp. 378-381.

[10] Y. Kim, L. Nan, J. Cong, and M. F. Chang, "High-Speed mm-Wave DataLink Based on Hollow Plastic Cable and CMOS Transceiver," IEEE Microwave and Wireless Components Letters, vol. 23, no. 12, pp. 674676, Dec. 2013.

[11] N. Van Thienen, Y. Zhang, and P. Reynaert, "Bidirectional Communication Circuits for a 120-GHz PMF Data Link in 40-nm CMOS," IEEE Journal of Solid-State Circuits, vol. 53, no. 7, pp. 2023-2031, Jul. 2018.

[12] N. V. Thienen, W. Volkaerts, and P. Reynaert, "A Multi-Gigabit CPFSK Polymer Microwave Fiber Communication Link in $40 \mathrm{~nm}$ CMOS," IEEE Journal of Solid-State Circuits, vol. 51, no. 8, pp. 1952-1958, Aug. 2016.

[13] M. Hitzler, S. Saulig, L. Boehm, W. Mayer, and C. Waldschmidt, "MMIC-to-Waveguide Transition at $160 \mathrm{GHz}$ with Galvanic Isolation," in IEEE MTT-S International Microwave Symposium (IMS), May 2016, pp. 1-4.

[14] N. Van Thienen, Y. Zhang, M. De Wit, and P. Reynaert, “An 18 Gbps Polymer Microwave Fiber (PMF) Communication Link in $40 \mathrm{~nm}$ CMOS," in 42nd European Solid-State Circuits Conference, Sep. 2016, pp. 483-486.

[15] J. Hasch, U. Wostradowski, S. Gaier, and T. Hansen, "77 GHz Radar Transceiver with Dual Integrated Antenna Elements," in German Microwave Conference Digest of Papers, Mar. 2010, pp. 280-283. 